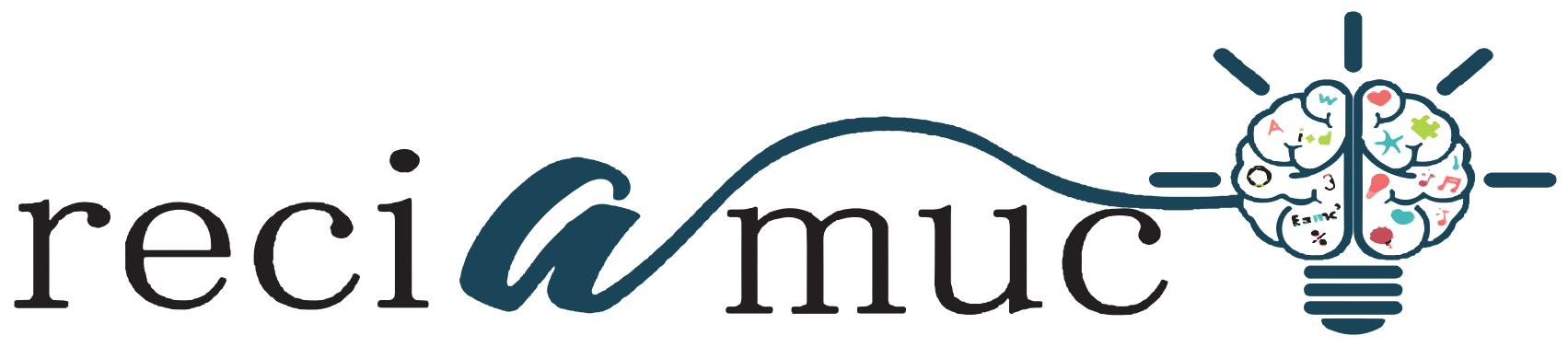

DOI: 10.26820/reciamuc/5.(4).noviembre.2021.232-244

URL: https://reciamuc.com/index.php/RECIAMUC/article/view/746

EDITORIAL: Saberes del Conocimiento

REVISTA: RECIAMUC

ISSN: 2588-0748

TIPO DE INVESTIGACIÓN: Artículo de Revisión

CóDIGO UNESCO: 32 Ciencias Médicas

PAGINAS: $232-244$

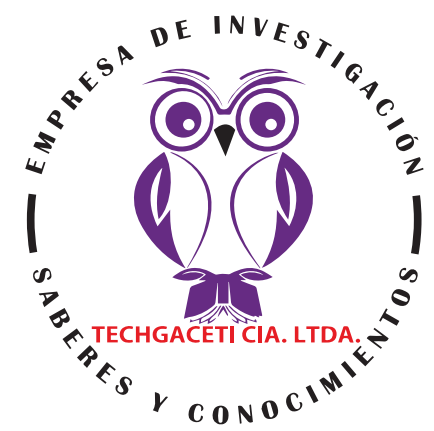

\title{
Consumo de alcohol durante la pandemia COVID-19 en América Latina y el Caribe
}

Alcohol consumption during the COVID-19 pandemic in Latin America and the Caribbean

Consumo de álcool durante a pandemia de COVID-19 na América Latịna

e no Caribe

Blanca Azucena Villacis Pérez'; Daniela Soledad Camacho Martínez;;

Franklin Emilio Narváez Cevallos; Camacho Martínez Adriana Belén ${ }^{4}$

RECIBIDO: 15/09/2021 ACEPTADO: 05/10/2021 PUBLICADO: 29/11/2021

1. Médico; Investigador Independiente; Guayaquil, Ecuador; bavp2305@gmail.com; (D) https://orcid.org/00000001-5196-0683

2. Médico; Investigador Independiente; Guayaquil, Ecuador; danielacamacho10@icloud.com; (iD https://orcid. org/0000-0002-4923-6407

3. Médico; Investigador Independiente; Guayaquil, Ecuador; emilionc.91@gmail.com; (DD https://orcid.org/00000001-6003-6484

4. Médico; Investigador Independiente; Guayaquil, Ecuador; dra.adrianacmartinez@gmail.com; (D) https://orcid. org/0000-0002-5111-3668

\section{CORRESPONDENCIA \\ Blanca Azucena Villacis Pérez \\ bavp2305@gmail.com \\ Guayaquil, Ecuador}

○ RECIAMUC; Editorial Saberes del Conocimiento, 2021 


\section{RESUMEN}

Los adultos y niños en situaciones de emergencia padecen una gran variedad de problemas mentales y neurológicos y de trastornos relacionados con el uso de sustancias psicoactivas. La mayoría siente pesadumbre y dolor psíquico agudo, que se consideran respuestas psicológicas normales y pasajeras a situaciones muy adversas. En los estudios de ámbito nacional en la Región de las Américas se describe un aumento del desasosiego, la depresión, la ansiedad y el insomnio, entre otros, como resultado de la pandemia de COVID-19 Con la finalidad de conocer las repercusiones en la población de América Latina y el Caribe se ha realizado una investigación acerca del consumo de alcohol a fin de conocer la situación actual en la región y en dado caso exponer el nivel de interés y atención que se ha de prestar para enfrentar una consecuencia mas de la pandemia con la COVID-19.Aun no hemos terminado de conocer los efectos de la COVID-19, la aparición de variantes y las modificaciones en la etiología de la enfermedad nos mantiene en alerta por tanto también es muy temprano conocer completamente cuales son los efectos emocionales que se reflejan en la población. América Latina y el Caribe tiene unas características económicas, políticas, culturales y sociales particulares que deben ser estudiadas con la rigurosidad que merece el caso, hasta los momentos los estudios disponibles en español en cuando al consumo de alcohol y las implicaciones psico-emocionales padecidas por la pandemia de la COVID-19 se limitan a México principalmente, Argentina y Brasil. Ha de ser menester de los procesos investigativos diseñar los instrumentos que revelen un mapa mas exacto de la situación en la actualidad en donde se puede tomar como apoyo los estudios realizados en Asia y Europa quienes demuestran atención investigativa hacia el tema.

Palabras clave: Depresión, consumo de alcohol, enfermedades emocionales, pandemia, COVID-19.

\section{ABSTRACT}

Adults and children in emergency situations suffer from a wide variety of mental and neurological problems and disorders related to the use of psychoactive substances. Most experience grief and acute psychic pain, which are considered normal and temporary psychological responses to very adverse situations. National studies in the Region of the Americas describe an increase in restlessness, depression, anxiety and insomnia, among others, as a result of the COVID-19 pandemic In order to know the repercussions on the population In Latin America and the Caribbean, an investigation has been carried out on alcohol consumption in order to know the current situation in the region and, if necessary, expose the level of interest and attention that must be paid to face one more consequence of the pandemic with COVID-19 We have not yet finished knowing the effects of COVID-19, the appearance of variants and modifications in the etiology of the disease keeps us on alert, therefore it is also very early to fully know what the emotional effects are that are reflected in the population. Latin America and the Caribbean have particular economic, political, cultural and social characteristics that must be studied with the rigor that the case deserves, until now the studies available in Spanish regarding alcohol consumption and the psycho-emotional implications suffered by the pandemic of COVID-19 are mainly limited to Mexico, Argentina and Brazil. It must be necessary for the investigative processes to design the instruments that reveal a more exact map of the current situation, where the studies carried out in Asia and Europe that demonstrate investigative attention to the subject can be taken as support.

Keywords: Guillain Barre syndrome, paralytic neuropathy, acute poly-neuropathy, COVID-19, flaccid paralysis.

\section{RESUMO}

Adultos e crianças em situações de emergência sofrem de uma grande variedade de problemas e transtornos mentais e neurológicos relacionados ao uso de substâncias psicoativas. A maioria experimenta luto e dor psíquica aguda, que são consideradas respostas psicológicas normais e temporárias a situações muito adversas. Estudos nacionais na Região das Américas descrevem aumento da inquietação, depressão, ansiedade e insônia, entre outros, em decorrência da pandemia COVID-19. Para conhecer as repercussões na população da América Latina e Caribe, uma investigação foi realizada sobre o consumo de álcool com o objetivo de conhecer a situação atual da região e, se necessário, expor o nível de interesse e atenção que se deve ter para enfrentar mais uma consequência da pandemia com COVID-19. conhecendo os efeitos do COVID-19, o surgimento de variantes e modificações na etiologia da doença nos mantém em alerta, pois também é muito cedo para sabermos plenamente quais são os efeitos emocionais que se refletem na população. A América Latina e o Caribe apresentam particularidades econômicas, políticas, culturais e sociais que devem ser estudadas com o rigor que o caso merece, até agora os estudos disponíveis em espanhol sobre o consumo de álcool e as implicações psicoemocionais sofridas pela pandemia de COVID- 19 estão principalmente limitados ao México, Argentina e Brasil. Deve ser necessário que os processos investigativos desenhem instrumentos que revelem um mapa mais exato da situação atual, onde possam ser tomados como suporte os estudos realizados na Ásia e na Europa que demonstrem atenção investigativa ao assunto.

Palavras-chave: Depression, alcohol consumption, emotional illnesses, pandemic, COVID-19. 


\section{Introducción}

Los adultos y niños en situaciones de emergencia padecen una gran variedad de problemas mentales y neurológicos y de trastornos relacionados con el uso de sustancias psicoactivas. La mayoría siente pesadumbre y dolor psíquico agudo, que se consideran respuestas psicológicas normales y pasajeras a situaciones muy adversas. Sin embargo, en una fracción pequeña de la población, estas situaciones desencadenan problemas de salud mental, tales como el estado depresivo, el trastorno por estrés postraumático y el trastorno por duelo prolongado, que pueden dificultar enormemente el desempeño de las actividades cotidianas. Además, las personas con trastornos graves preexistentes, como psicosis, discapacidad intelectual y epilepsia, se tornan aún más vulnerables debido al desplazamiento, al desamparo y a la ausencia de servicios de salud. Por último, el consumo de alcohol y drogas plantea graves riesgos en materia de salud y de violencia de género. Si, por un lado, las necesidades de salud mental de la población aumentan ostensiblemente, por el otro muchas veces se carece de recursos locales para atenderlas. En tales circunstancias se necesitan, más que nunca, herramientas prácticas y fáciles de usar. (OPS, 2016)

En 12 de diciembre de 2019, la Comisión Municipal de Salud de Wuhan, en la República Popular de China, hizo público un reporte de 27 casos de personas con una neumonía viral. El Centro Chino para el Control y la Prevención de Enfermedades (CCDC) identificó un nuevo beta-coronavirus denominado 2019-nCoV, siendo nombrado oficialmente por la Organización Mundial de la Salud (OMS) como enfermedad por coronavirus (Covid-19) el 11 de febrero de 2020, convirtiéndose así en una amenaza global por su rápida propagación, y un problema de salud pública. El 11 de marzo de 2020, la OMS decreta el estado de pandemia. A nivel social, las medidas adoptadas pueden conducir a trastornos psicológicos y psi- quiátricos por factores como separación de la familia, dolor, duelo, soledad, vergüenza, culpa, ira, miedo, xenofobia, histeria colectiva, desinformación en las redes sociales, inseguridad financiera, la estigmatización y otros. (Apaza P., Seminario Sanz, \& Santa-Cruz Arévalo, 2020)

La información relevante respecto al impacto psicológico del confinamiento debido a la pandemia por el nuevo coronavirus (SARSCov-2) emplea conceptos de cuarentena y confinamiento, así como de aislamiento y distanciamiento social, es necesario distinguir cada uno de ellos con el propósito de contribuir a su correcto uso entre especialistas de las ciencias de la salud. Estos conceptos denotan medidas de contención para evitar la propagación de una enfermedad contagiosa. La cuarentena se aplica ante la sospecha de que una persona o un grupo de personas están infectadas, mientras que el aislamiento se da cuando se ha confirmado el contagio. El distanciamiento social significa separarse y mantener un espacio físico determinado entre individuos, en cambio, el confinamiento es un plan de intervención comunitario que implica permanecer refugiado el mayor tiempo posible, bajo nuevas normas socialmente restrictivas. (Sánchez-Villena \& Fuente de la, 2020)

La cuarentena es una medida preventiva necesaria durante epidemias relacionadas con algunas enfermedades importantes, pero puede tener un impacto psicológico negativo amplio y sustancial en la población expuesta. Esto sugiere la necesidad de integrar medidas efectivas que mitiguen este impacto como parte del proceso de planificación de la misma. La complejidad de esta medida supone un desafío para los responsables políticos, y una oportunidad para la realización de revisiones rápidas que sinteticen la evidencia disponible para planificar, desarrollar e implementar políticas de salud oportunas, eficaces y adecuadas a las circunstancias incluso recomendadas por la OMS. (García Ron \& Cuéllar Flores, 2020) 
Al 2 de noviembre del 2020, en la Región de las Américas se han registrado 20.733.940 casos confirmados de COVID-19 y 642.995 muertes asociadas a esta enfermedad. Desde junio del 2020, la Región de las Américas ha estado en el epicentro de la pandemia de COVID-19. Hasta el 31 de agosto del 2020, seis de los diez países con un mayor número de casos notificados a nivel mundial se encontraban en el continente, si bien las tendencias actuales muestran una disminución tanto en lo que respecta al número de casos como en el número de muertes. En los estudios de ámbito nacional en la Región de las Américas se describe un aumento del desasosiego, la depresión, la ansiedad y el insomnio, entre otros, como resultado de la pandemia de COVID-19. (OPS, 2020)

El miedo, la preocupación y el estrés son respuestas normales en momentos en los que nos enfrentamos a la incertidumbre, o a lo desconocido o a situaciones de cambios o crisis. Así que es normal y comprensible que la gente experimente estos sentimientos en el contexto de la pandemia COVID-19. Ante las nuevas y desafiantes realidades de distanciamiento físico, el trabajo desde el hogar, el desempleo temporal, la educación de los niños en el hogar y la falta de contacto físico con los seres queridos y amigos, es importante que cuidemos tanto nuestra salud física como mental. La OPS/OMS, junto con sus colaboradores, está brindando orientación y asistencia a la Región de las Américas durante la pandemia COVID-19, para ayudar a apoyar el bienestar mental y general de todas las personas, especialmente de aquellos que podrían necesitar apoyo adicional durante estos difíciles momentos. (OPS, 2021)

Para comprender la repercusión de la pandemia en los servicios para los trastornos mentales, neurológicos y por consumo de sustancias psicoactivas, la Organización Mundial de la Salud (OMS) elaboró una encuesta, que fue realizada por la OMS y la Organización Panamericana de la Salud
(OPS) y se envió a los puntos focales de salud mental designados para ello en los ministerios de salud de todos los Estados Miembros de la OMS. La encuesta evaluaba la existencia y el financiamiento de planes para prestar servicios esenciales de salud mental y apoyo psicosocial, la presencia y composición de las plataformas de coordinación de estos servicios, el grado de continuidad y las causas de las interrupciones en los diferentes servicios de este tipo, los enfoques usados para abordarlas y los mecanismos de vigilancia, así como la investigación sobre los datos de estos trastornos. (OPS, 2020)

La prevalencia del consumo nocivo de alcohol o de drogas puede aumentar durante las crisis humanitarias debido a que en ellas los adultos y los adolescentes a menudo recurren a la automedicación para sobrellevar el estrés, las pérdidas y el dolor. Las situaciones de emergencia pueden ocasionar una reducción del abastecimiento de alcohol y de drogas, lo cual explica por qué aparecen, de manera repentina, síntomas de abstinencia potencialmente mortales en personas que han consumido sustancias durante mucho tiempo a dosis relativamente altas. Esto sucede especialmente en casos de consumo de alcohol. (Organización Panamericana de la Salud, 2016)

El alcohol es un gran factor de riesgo de mortalidad y morbilidad en las Américas y es responsable del 6,5\% de todas las muertes en la región. Datos emergentes de varios informes clave destacan el coste social y económico del consumo nocivo de alcohol, las principales estrategias utilizadas por la industria del alcohol durante la pandemia, y las formas y el grado en que los incentivos otorgados a las mismas industrias acaban yendo en contra de los objetivos de salud pública y seguridad. (OPS, 2021)

El Departamento de Enfermedades No Transmisibles y Salud Mental llevó a cabo el seminario web "Alcohol y COVID-19 en las Américas: ¿qué ha pasado y qué hacemos 
para avanzar?", el 30 de agosto de 2021 en el cual aborda los cambios en los patrones de consumo de alcohol de diversas maneras con el objetivo de revisar la situación del consumo de alcohol, las políticas y las respuestas en las Américas en el contexto de la COVID-19, y discutir lo que se puede hacer a medida que avanzamos para reducir el uso nocivo del alcohol y construir sistemas de salud más resistentes mientras seguimos lidiando con la pandemia de la COVID-19 (OPS, 2021)

El abordaje de los factores psicosociales presentes durante la pandemia por el Covid-19, requieren de estudios a profundidad y en muestras más amplias, considerando diferentes segmentos de la población y de diferentes ámbitos geográficos, a fin de contribuir a intervenciones efectivas por medio de política públicas de salud mental en situaciones de confinamiento, denotando que la salud física es tan importante como la salud mental. (Apaza P., Seminario Sanz, \& Santa-Cruz Arévalo, 2020)

Con la finalidad de conocer las repercusiones en la población de América Latina y el Caribe se ha realizado una investigación acerca del consumo de alcohol a fin de conocer la situación actual en la región y en dado caso exponer el nivel de interés y atención que se ha de prestar para enfrentar una consecuencia mas de la pandemia con la COVID-19.

\section{Metodología}

Se trata de un estudio bibliográfico descriptivo longitudinal de las publicaciones científicas disponibles a la fecha que refiera la situación actual del desarrollo emocional y físico experimentado por la población durante la pandemia ocasionada la enfermedad de COVID-19 y la repercusión en el consumo de alcohol en América Latina y el Caribe.

Para la consecución del objetivo se ha considerado no solo el consumo de alcohol como variable para la selección de la infor- mación sino todos la implicaciones sociales, emocionales y psicológicas que desencadenan la necesidad de cierta población al consumo de sustancias adictivas, entre las que se encuentra el alcohol.

La información recabada en índices como PUBMed, Scielo, Google Académico, Elseiver entre páginas de Organismos oficiales han sido los elementos principalmente considerados para la selección de los trabajos cuyo análisis, interpretación, correlación y resumen se presentan a continuación.

\section{Resultados}

Las drogas que causan depresión de conciencia pueden comprometer el aparato respiratorio por complicación aspirativa. Las benzodiacepinas, como ansiolíticas, sedantes o hipnóticas, pueden ser utilizadas bajo forma de automedicación y aumentar el riesgo de sobredosis, solas o asociadas a la ingesta de alcohol. Otras drogas depresoras, como los opiáceos y opioides, pueden ocasionar depresión respiratoria, incrementando la morbimortalidad en caso de COVID-19. La desinhibición causada por efecto del consumo de alcohol puede asociarse a conductas de riesgo que incrementen el contagio o comprometan el autocuidado. Algo similar ocurre con el consumo de drogas perturbadoras, disociativas o alucinógenas. El consumo crónico de alcohol y cocaína se ha relacionado con enfermedades cardiovasculares y alteraciones de la inmunidad que han demostrado incrementar la morbimortalidad por COVID-19. (Pascale, 2020)

El consumo de alcohol o drogas, y diversos medicamentos sujetos a prescripción médica, puede dar origen a diversos problemas. Entre ellos se encuentran el síndrome de abstinencia (síntomas físicos y mentales que aparecen cuando la sustancia se suspende o se reduce bruscamente), la dependencia y el consumo nocivo (con daño a la salud física o mental o al bienestar general). El consumo de alcohol o drogas es nocivo cuando causa trastornos físicos o mentales, 
comportamientos de riesgo para la salud, problemas familiares o conyugales, violencia sexual y física, accidentes, maltrato y negligencia de menores, dificultades económicas y otros problemas ligados a la desprotección. (Organización Panamericana de la Salud, 2016)

En condiciones normales, en un contexto de no aislamiento ni confinamiento, el estrés puede ser un potencial detonante tanto para el inicio como para el mantenimiento del consumo problemático de alcohol. De la misma manera, el consumo crónico de alcohol produce neuroadaptaciones en las vías de estrés y recompensa, que conducen a ejes disfuncionales caracterizados por una desregulación de la respuesta al cortisol y déficits en la regulación emocional. (Sixto-Costoya, Lucas-Domínguez, \& Agulló-Calatayud, 2021)

El consumo excesivo de Alcohol (CEA) corresponde al consumo de cinco o más copas en una ocasión en el caso de los hombres, o cuatro o más copas en una ocasión en el caso de las mujeres, esto con una frecuencia diaria, semanal, mensual u ocasional (1 a 11 veces al año). Una copa o "unidad de medida estándar" corresponde a lo siguiente: una botella, lata o vaso de cerveza; un coctel "paloma" o "coolers"; una copa de vino o licor; un "caballito" de tequila o mezcal; un vaso de brandy, ron o whisky o un "jarrito" de pulque. La intensidad de este patrón de consumo está medida en el número de copas consumidas por ocasión y la frecuencia en la periodicidad de ocurrencia del evento: diario, semanal, mensual, ocasional o nunca. (Rivera-Rivera, Séris-Martínez, Reynales-Shigematsu, Villalobos, \& Jaen-Cortés, 2021)

Hallazgos relacionados al consumo de alcohol por la población en medio de la pandemia.

En un estudio realizado en Argentina respecto al temor asociado al COVID-19 en se encuentra que fue menor en comparación al reportado en la población iraní y similar al hallado en la población italiana. Esto podría deberse a cuestiones culturales, o bien a la percepción de una mayor sensación de cuidado en la población argentina, como efecto de las fuertes medidas adoptas tempranamente por el gobierno, a diferencia de las situaciones de Irán y de Italia. En este estudio los jóvenes fueron quienes manifestaron mayor consumo de alcohol desde el inicio de la cuarentena, hecho que podría estar estrechamente relacionado con los sentimientos de tristeza y de soledad. Los hallazgos del presente estudio resultan de utilidad a fin de determinar grupos de riesgo y/o de vulnerabilidad en la población, con el objetivo de implementar intervenciones tempranas de prevención y de tratamiento durante la presente situación de emergencia y en las próximamente inmediatas, cuando comience a cesar el aislamiento obligatorio. (Alomo, y otros, 2020)

En el caso del SARS, el abuso o la dependencia del alcohol fue vinculados al trabajo en el sector de la salud durante la epidemia, incluso tres años después del brote (Wu et al., 2008). Una mayor grado de exposición al virus y tener que ser aislado como consecuencia se revelaron como factores de riesgo, se identificó una relación dosis-respuesta entre la intensidad del virus exposición y síntomas de abuso de alcohol a largo plazo y dependencia En cuanto a la pandemia COVID-19, para el momento de investigación, año 2020, en España aún no existen datos sobre trastornos por abuso de sustancias o estudios que evalúen el posible aumento del consumo como consecuencia de encierro. (García, Fuente de la, Sáiz, García, \& Bobes, 2020)

Un reciente estudio llevado a cabo en China con el objetivo de averiguar los problemas psicológicos asociados a la situación derivada de la COVID-19 y del aislamiento masivo, concluye que existe un aumento de la tasa de ansiedad y depresión, un consumo de alcohol peligroso y dañino, y una relación de bienestar mental inferior a la habitual. Según este estudio, la franja de edad 
más afectada en términos de salud mental y consumo de alcohol es la de 21 a 40 años (Ahmed et al., 2020). (Sixto-Costoya, Lucas-Domínguez, \& Agulló-Calatayud, 2021)

En una línea parecida, Lee (2020) utiliza el nuevo instrumento Coronavirus anxiety scale (CAS) para evaluar la ansiedad disfuncional asociada con la crisis de la COVID-19 en el contexto de EE.UU. Estos autores detectaron que mayores niveles de ansiedad, peor diagnóstico de COVID-19, aumento de consumo de alcohol y otras drogas, generan incapacidad extrema para determinadas actividades e ideación suicida. (Sixto-Costoya, Lucas-Domínguez, \& Agulló-Calatayud, 2021)

Reynolds \& Wilkinson (2020) advierten desde el contexto australiano del peligro de considerar las tiendas donde se vende alcohol como de primera necesidad, es decir, que se mantienen abiertas en la pandemia. Según ellos, esto conlleva a un posible aumento de alcohol en los hogares que traería complicaciones tanto de convivencia como de favorecimiento de conductas adictivas. (Sixto-Costoya, Lucas-Domínguez, \& Agulló-Calatayud, 2021)

En otra línea totalmente distinta, un trabajo llevado a cabo desde el contexto iraní alerta sobre los graves daños que está causando en la población la desinformación referente al consumo de alcohol, debido a la expansión de información no basada en ningún tipo de evidencia científica que afirma que la ingesta de alcohol detiene la expansión del virus SARS-CoV2. Esta creencia se debe, según indican los autores, a factores culturales vinculados con la prohibición del alcohol lo que conlleva una falta de formación e información al respecto. (Sixto-Costoya, Lucas-Domínguez, \& Agulló-Calatayud, 2021)

En México, en cuanto al nivel de consumo de alcohol se identificó que un alto porcentaje de la población universitaria bebe de manera perjudicial, la cifra es alarmante ya que puede convertirse en una dependen- cia. Han sido caracterizados altos daños en materia de adicciones y salud mental en el alumnado, sobretodo el que corresponde al área de salud y medicina, teniendo como principal detonante de la ingesta de alcohol la temprana edad en que es probada por primera vez esta sustancia. (López Posadas, Gómez Rodríguez, Sibaja Reyes, \& Marín Gómez, 2021)

Es verdad que se produjo un aumento de consumo de alcohol en las primeras semanas de confinamiento Smith (2020) para luego decrecer según avanza el periodo de encierro y permanencia con la familia, según Newby (2020) los dos autores tienen razón al estar una persona confinada y tener niveles altos de estrés el aumento de consumo de alcohol se dio en las primeras semanas pero disminuyo con el tiempo. Las condiciones del confinamiento son una experiencia traumática, que producen tensiones fuertes y persistentes, que producen una debilitación crónica u estrés continuo (TEPT), que estaría asociado al consumo de alcohol. (Andrade Chauvin, 2021)

Es necesario estar preparados para la atención psicológica, elemento que fue necesario para dar una primera respuesta emocional a la población general, a esto indica, que generalmente en un desastre el personal de salud, incluidos los psicólogos no está preparados en técnicas de Atención psicológica en emergencias, o Primeros Auxilios Psicológicos (PAS), segundo, los trabajadores de la salud mental son reclutados de varias áreas de trabajo y están poco preparados para su labor en emergencias, así tratan de usar los pocos conocimientos sobre manejo de emociones en desastres, trabajando mucho y descansando poco, lo que causa un desgaste, que podría producir a largo plazo también altos niveles de ansiedad, miedo y estrés y tercero, el personal de salud que está sobreexpuesto al sufrimiento y el dolor está en constante estrés, convirtiéndose en el grupo más vulnerable. (Andrade Chauvin, 2021) 
Las limitaciones propias de estar en un momento histórico, suigéneris y único que no ha permitido el normal desarrollo de las actividades y causo una crisis en todos los ámbitos, provocando la subestimación o sobrestimación de la prevalencia de estrés y del consumo de alcohol, al no poder contar con estudios poblacionales amplios. El consumo de alcohol es directa e inversamente proporcional al avance de la pandemia. El inicio del confinamiento produjo una crisis existencial, la misma que altera la afectividad y emotividad produciendo inestabilidad y ansiedad, lo que a su vez provoca un aumento de consumo de alcohol en los dos primeros meses de encierro. El confinamiento con la familia es un elemento protector del consumo de alcohol. (Andrade Chauvin, 2021)

La principal conclusión es la marcada incidencia e interrelación de las medidas para afrontar la COVID-19 y sus consecuencias directas en la salud mental y las adicciones. La mayoría de los estudios se están centrando en consumidores de opiáceos, tabaquismo y alcohol. En relación con los primeros se constatan afecciones psiquiátricas comórbidas, que podrían aumentar las sobredosis, muy especialmente, entre aquellas personas que sufren situaciones de marginalidad y estigma. El consumo problemático de alcohol, se relacionaría con el estrés, pudiendo erigirse por tanto en un potencial detonante tanto para el inicio como para el mantenimiento de los consumos -especialmente en concurrencia con aislamiento. (Sixto-Costoya, Lucas-Domínguez, \& Agulló-Calatayud, 2021)

En estudios previamente publicados, se han reportado cambios en la ingesta de alimentos durante el confinamiento y se observaron patrones alimentarios no saludables, tales como el incremento en el consumo de refrigerios, consumo de alimentos con alto índice glucémico y mayor ingesta de alimentos ultraprocesados. Estos cambios se han observado en todos los grupos etarios, y ha sido mayormente visible en sectores con desigualdades sociales importantes. No solo la ingesta de alimentos se ha visto modificada, sino también se ha reportado un incremento en el consumo de alcohol y tabaco (Almendra, y otros, 2021);

El aumento en el consumo de alcohol en México solo fue del $9.3 \%$ vs. $31 \%$ reportado por las muestras estadounidenses. Probablemente, la disminución percibida por parte de los participantes se deba a las políticas de restricción aplicadas a la industria del alcohol durante la fase de la pandemia, así como la puesta en marcha de la llamada ley seca o prohibición de venta de bebidas con graduación alcohólica durante la Fase 3 de la pandemia. Aunque también puede estar relacionado con las características de la muestra, ya que la mayoría refirió un consumo responsable e incluso la no ingesta de alcohol. (Carrillo Valdez, Reyes Muñiz, Escamilla Gutiérrez, \& González Baena, 2021)

CEA es prevalente en México hasta noviembre del 2020. Los principales hallazgos de este estudio mostraron una asociación positiva entre el CEA y los siguientes factores: sexo (hombre), edad (adultos jóvenes), escolaridad (alta), situación laboral (trabajar durante el confinamiento), presencia de personas en edad escolar en el hogar (uno o más) y algunas regiones geográficas. El estudio mostró que casi la mitad de la población ha consumido alcohol de manera excesiva y es más frecuente en hombres que en mujeres. Estos resultados son similares a estudios previos realizados en la Región de las Américas y Brasil. (Rivera-Rivera, Séris-Martínez, Reynales-Shigematsu, Villalobos, \& Jaen-Cortés, 2021)

En México, los resultados de diferentes encuestas nacionales coinciden con los hallazgos, un estudio reciente encontró una fuerte asociación de CEA en los hombres. A pesar de que existe mayor riesgo de CEA en hombres que en mujeres, recientemente este tipo de consumo ha incrementado principalmente en las jóvenes quienes tienen 
mayor riesgo de CEA. Además se encontró que los adultos jóvenes (30 a 39 años) tuvieron hasta tres veces más posibilidad de CEA durante la pandemia que los de mayor edad.Datos similares se encontraron en estudios realizados en Italia y Bélgica. (Rivera-Rivera, Séris-Martínez, Reynales-Shigematsu, Villalobos, \& Jaen-Cortés, 2021)

En el presente estudio se encontró que las personas que tienen un nivel de escolaridad y socioeconómico alto tienen mayor posibilidad de CEA, principalmente en las mujeres. La situación conyugal se asoció con un mayor CEA, pues aquellas personas casadas/unidas y separadas/ divorciadas o viudas tuvieron una mayor posibilidad de CEA, principalmente en hombres. Estos hallazgos difieren de los encontrados en otros países donde ser soltero incrementa el riesgo de consumo de alcohol. (Rivera-Rivera, Séris-Martínez, Reynales-Shigematsu, Villalobos, \& Jaen-Cortés, 2021)

Un hallazgo relevante del presente estudio fue la asociación de CEA con la actividad laboral durante el confinamiento por la pandemia Covid-19, siendo de mayor riesgo entre aquellos que trabajaron desde casa, y los que salieron a trabajar todos los días. Este resultado es consistente con el estudio de Vanderbruggen y colaboradores y un estudio realizado en Noruega. La incertidumbre económica combinada con el trabajo desde casa debido a la pandemia Covid-19 son eventos estresores que exacerban el consumo de alcohol. (Rivera-Rivera, Séris-Martínez, Reynales-Shigematsu, Villalobos, \& Jaen-Cortés, 2021)

Se encontró que el tener al menos una persona en edad escolar en casa durante el periodo de pandemia estuvo asociado con el CEA, principalmente en hombres. Este hallazgo es similar al reportado por WardeII y colaboradores. Los cierres de escuelas y guarderías por la pandemia ocasionaron mayor carga a la familia, lo que incrementó el consumo de alcohol como medida de afrontamiento, principalmente en hombres
Se encontró que en ciertas regiones del país como Pacífico-Norte y Sur, la Ciudad de México y zonas urbanas, existe una mayor posibilidad de CEA. Estos datos son similares a encuestas previas realizadas en México y al estudio de Ferrante y colaboradores al encontrar mayor posibilidad de CEA en las zonas urbanas.. (Rivera-Rivera, Séris-Martínez, Reynales-Shigematsu, Villalobos, \& Jaen-Cortés, 2021)

La OMS ha identificado el consumo abusivo de alcohol como uno de los factores de riesgo en la violencia contra la pareja y la violencia sexual, incrementando las probabilidades de que las agresiones sean más graves. También, que las mujeres que han sufrido violencia de pareja tienen casi el doble de posibilidades de padecer problemas con el alcohol. Estas relaciones cobran especial relevancia en la situación de confinamiento por COVID-19 durante la cual han aumentado los índices de violencia de género. (Villanueva, y otros, 2021)

Con base en los resultados se propone una estrategia de intervención psicosocial que incluya:

1. Psicoeducación sobre los efectos en la salud física, emocional, psicológica y social del consumo excesivo de comida y alcohol;

2. Desmitificación de creencias sobre los alimentos, el alcohol y COVID-19;

3. Técnicas de desactivación fisiológica para el manejo de los síntomas somáticos de ansiedad y estrés;

4. Activación conductual con el propósito de disminuir los periodos de aburrimiento a partir de actividades planificadas y valiosas para la vida;

5. Entrenamiento en solución de problemas para favorecer los estilos de afrontamiento orientados a la aproximación;

6. Entrenamiento en autocontrol, con la finalidad de contribuir a la disminución de 
conductas perjudiciales para la salud y

7. Auto reforzamiento, para que la persona sea capaz, por sí misma, de mantener los cambios generados. (Carrillo Valdez, Reyes Muñiz, Escamilla Gutiérrez, \& González Baena, 2021)

\section{El alcohol y la COVID-19 Lo que debe saber:}

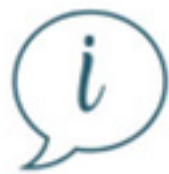

Bajo ninguna circunstancia debe consumir ningún tipo de bebidas alcohólicas como una manera de prevenir o tratar la COVID-19

El consumo de alcohol NO le protegerá frente a la COVID-19.

Evite por completo el alcohol

Evite por completo el alcohol para no periudicar su propio sistema irmunitario y su salud, ni poner en riesgo la salud de los demás.

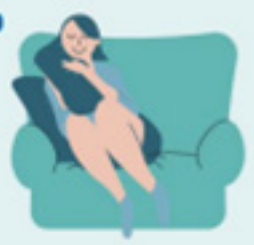

\section{Busque ayuda}

si cree que su consumo de alcohol o el de alguien cercano a usted está fuera de control.

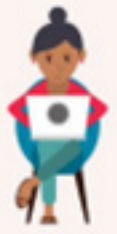

Evite que el alcohol le dé pie a fumar y viceversa, ya que el consumo de alcohol está asociado con una progresión maycr y más peligrosa de la CoviD-19

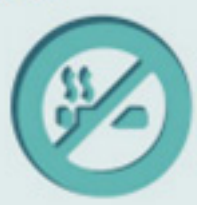

Hable con los niños, adolescentes y jóvenes sobre los problemas asociados con el consumo de alcohol y la COVID-19 como las violaciones a la cuarentena y el distanciamiento social, que pueden empeorar la pandemia.
No utilice el alcohol como una manera de enfrentar sus emociones y el estrés ya que estar aislado y beber alcohol también puede incrementar el riesgo de suicidio. Si tiene pensamientos suicidas, llame a una linea telefónica de ayuda.

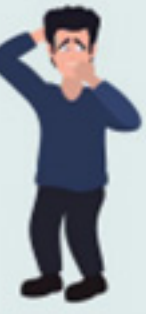

Nunca mezcle el alcohol con medicamentos

incluso aunque se trate de remedios naturales o sin receta, ya que esto puede reducir su efectividad o incrementar su potencia a un nivel que puede ser tóxico y peligroso.

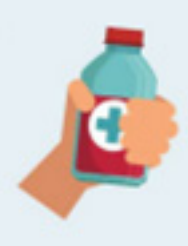

Asegúrese de que los niños, adolescentes y jóvenes no tengan acceso al alcohol y no beba en su presencia; sea un modelo para ellos. Controle el tiempo que sus hijos pasan ante la pantalla, ya que la televisión y coros medios están inundados de publicidad de alcohol y desinformación que pueden estimular un inicio temprano en el consumo.

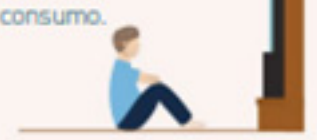

World Health

Organization

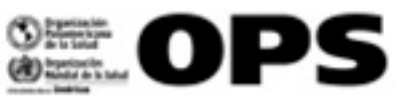

Cuadro 1. Folleto difundido por la OMS sobre el Alcohol y la COVID-19 Fuente: (OPS, 2021) 


\section{Conclusiones}

Las personas con problemas emocionales y que carecen de estrategias de afrontamiento funcionales (es decir, que les permitan una eficaz regulación emocional) tienden a desarrollar un comportamiento alimentario inadecuado, sustituyendo la regulación emocional por la alimentación, lo que conlleva a un aumento de peso. Dentro de los principales cambios evidenciados se observa el aumento en el consumo de bebidas alcohólicas, donde en situaciones de estrés, se le atribuye al alcohol la capacidad de reducir el estrés mental y permite mantener un estado de relajación tanto física como mental la salud mental y estado emocional han llevado a un desarrollo de mecanismos compensatorios como la preferencia de alimentos "reconfortantes" y al aumento en el consumo de alcohol. (Garzón rueda, 2021)

La ausencia de resultados concluyentes respecto al efecto de la situación de confinamiento por COVID-19 en el consumo de alcohol, justifica el desarrollo de nuevos estudios que permitan obtener mayor nivel de evidencia. (Villanueva, y otros, 2021)

Las estrategias de sensibilización y los programas de detección e intervención breve sobre el consumo de riesgo de alcohol deben diseñarse y dirigirse de acuerdo a la identificación de la población que ha padecido el CEA durante el confinamiento por la pandemia de la COVID-19.

La estabilidad laboral y la minimización de los efectos económicos y de desempleo que pueden asociarse a situaciones de crisis como la derivada del confinamiento por la COVID-19, son elementos a considerar en relación a la reducción del consumo de riesgo de alcohol. A este respecto, puede ser de interés la aplicación de medidas para reducir factores ambientales promotores de estrés que, asimismo, pueden generar un incremento del consumo de riesgo de alcohol en la población (Villanueva, y otros, 2021)
Un nuevo documento de la Organización Mundial de la Salud (OMS) pretende guiar la respuesta a nivel nacional y local, ofreciendo orientaciones para iniciar una transición progresiva para relajar las restricciones sobre el confinamiento a la vez que se mantiene la pandemia bajo control. Esta transición, debe basarse en la evidencia, apoyarse en datos y ser implementada gradualmente. En un nuevo comentario publicado en Evidencias COVID-19, en el que se analiza este documento, se resumen lo seis criterios que propone la OMS para alcanzar el levantamiento del confinamiento en los países. (The Cochrane Collaboration, 2021)

1. Control de la transmisión de la COVID-19 y número de casos nuevos

2. Cambio de orientación en la respuesta del sistema, de la identificación de casos graves a la detección y aislamiento de todos los casos (detección, realización de pruebas, aislamiento, cuarentena)

3. Riesgo de brotes en entornos de alta vulnerabilidad

4. Medidas preventivas en los puestos de trabajo

5. Gestión del riesgo de casos importados

6. Compromiso de toda la comunidad

Para reducir el riesgo de nuevos brotes, según la OMS, las medidas de restricción deben ser levantadas de forma escalonada y gradual, basándose en una evaluación de los riesgos epidemiológicos y de los beneficios socioeconómicos de levantar tales restricciones en diferentes lugares de trabajo, centros educativos y actividades sociales (como conciertos, eventos religiosos o deportivos). (The Cochrane Collaboration, 2021)

Disminuir las restricciones que se ha impuesto para frenar los contagios por el SARS-COV-2 es un objetivo multifactorial entre los que se incluye el esfuerzo de los 
elementos que componen la vida en comunidad. El distanciamiento social ha sido la única medida 100\% efectiva que evita la propagación del virus y por tanto el padecimiento de la COVID-19 sin embargo, paradójicamente es la medida que ha logrado impactar emocional y psicológicamente a la población sin distingo de sexo, cultura, edad o situación psicosocial.

Aun no hemos terminado de conocer los efectos de la COVID-19, la aparición de variantes y las modificaciones en la etiología de la enfermedad nos mantiene en alerta por tanto también es muy temprano conocer completamente cuales son los efectos emocionales que se reflejan en la población.

América Latina y el Caribe tiene unas características económicas, políticas, culturales y sociales particulares que deben ser estudiadas con la rigurosidad que merece el caso, hasta los momentos los estudios disponibles en español en cuando al consumo de alcohol y las implicaciones psicoemocionales padecidas por la pandemia de la COVID-19 se limitan a México principalmente, Argentina y Brasil.

Ha de ser menester de los procesos investigativos diseñar los instrumentos que revelen un mapa más exacto de la situación en la actualidad en donde se puede tomar como apoyo los estudios realizados en Asia y Europa quienes demuestran atención investigativa hacia el tema.

\section{Bibliografía}

Almendra, R., Baladia, E. R., Rojas, P., Vila, A., moya, J., Apolinar, E., . . Raggio, L. y. (2021). Conducta alimentaria durante el confinamiento por COVID-19 (CoV-Eat Project): protocolo de un estudio transversal en países de habla hispana. Revista de Nutrición Clínica y Metabolismo, 4(3), 150-156.

Alomo, M., Gagliardi, Georgina, Peloche, S., Eugenia Somers, E. S., Alzina, P., \& Prokopez, C. R. (2020). Efectos psicológicos de la pandemia covid-19 en la población general de Argentina. Revista de la Facultad de Ciencias Médicas de Córdoba, 77(3), 176-181.
Andrade Chauvin, W. (2021). El estrés y el consumo de alcohol, durante la pandemia de la covid-19. Quito: Universidad Central del Ecuador.

Apaza P., C., Seminario Sanz, R., \& Santa-Cruz Arévalo, J. (2020). Factores psicosociales durante el confinamiento por el Covid-19 - Perú. Revista Venezolana de Gerencia, 25(90), 402-410.

Carrillo Valdez, L., Reyes Muñiz, J. M., Escamilla Gutiérrez, M. L., \& González Baena, V. (2021). Conducta alimentaria y consumo de alcohol durante el distanciamiento social por COVID-19 en México: un estudio exploratorio. Ciencia y Sociedad, 46(2), 7-30.

García Ron, A., \& Cuéllar Flores, I. (2020). Impacto psicológico del confinamiento en la población infantil y como mitigar sus efectos: revisión rápida de la evidencia. Anales de Pediatría, 93(1), 57-58.

García, L., Fuente de la, L., Sáiz, P., García, M., \& Bobes, J. (2020). Will changes in alcohol and tobacco use be seen during the COVID-19 lockdown? Adicciones, 32(2), 85-89.

Garzón rueda, M. (2021). Análisis de los cambios en el consumo de alimentos y psicología ndividual en adultos que favorecen el desarrollo de ambiente obesogénico como consecuencia del confinamiento por covid-19 reportados en literatura científica mundial. Bogotá: Pontificia Universidad Javeriana

López Posadas, J. R., Gómez Rodríguez, J., Sibaja Reyes, I., \& Marín Gómez, L. S. (2021). Depresión y consumo de alcohol en estudiantes universitarios de enfermería durante el confinamiento por Covid-19. Jóvenes en la Ciencia, https://www.jovenesenlaciencia.ugto.mx/index.php/jovenesenlaciencia/article/view/3253.

OPS. (2016). Guía de intervención humanitaria mhGAP (GIH-mhGAP). El manejo clínico de los trastornos mentales. Washington, DC: Organización Panamericana de la Salud.

OPS. (2020). La repercusión de la COVID-19 en los servicios para los trastornos mentales, neurológicos y por consumo de sustancias psicoactivas en la Región de las Américas: resultados de una evaluación rápida. Washington, D.C: Organización Panamericana de la Salud.

OPS. (2021). Alcohol y COVID-19 en las Américas: ¿qué ha pasado y qué hacemos para avanzar? Obtenido de Organización PAnamericana de la Salud: https://www.paho.org/es/eventos/alcohol-covid-19-americas-que-ha-pasado-que-hacemos-para-avanzar 
OPS. (2021). Salud mental y Covid-19. Obtenido de Organización Panamericana de la Salud: https:// www.paho.org/es/salud-mental-covid-19

Organización Panamericana de la Salud. (2016). Guía de intervención humanitaria mhGAP (GIH-mhGAP). El manejo clínico de los trastornos mentales neurológicos y por uso de sustancias en las emergencias humanitarias. Washington, D.C: Organización Panamericana de la Salud.

Pascale, A. (2020). Consumo de sustancias psicoactivas durante la pandemia por COVID-19: implicancias toxicológicas desde un enfoque integral y desafíos terapéuticos. Revista Médica del Uruguay, 36(3).

Rivera-Rivera, L., Séris-Martínez, M., Reynales-Shigematsu, L., Villalobos, A., \& Jaen-Cortés, C. N.-R. (noviembre-diciembre de 2021). Factores asociados con el consumo excesivo de alcohol: Encuesta Nacional de Salud y Nutrición 2020 sobre Covid-19. Salud Pública de México, 65(6), 789-798.
Sánchez-Villena, A., \& Fuente de la, V. (julio de 2020). COVID-19: cuarentena, aislamiento, distanciamiento social y confinamiento, ¿son lo mismo? 93(1), 73-74.

Sixto-Costoya, A., Lucas-Domínguez, R., \& AguIló-Calatayud, V. (2021). COVID-19 y su repercusión en las adicciones. Health and Addictions / Salud y Drogas, 21(1), 259-278.

The Cochrane Collaboration. (2021). Medidas de la OMS para levantar el confinamiento. Obtenido de Cochrane Iberoamérica: https://es.cochrane.org/ es/news/medidas-de-la-oms-para-levantar-el-confinamiento

Villanueva, V., Motos, P., Isorna, M., Villanueva, V., Blay, P., \& Vázquez, A. (2021). Impacto de las medidas de confinamiento durante la pandemia de Covid-19 en el consumo de riesgo de alcohol. Revista Española Salud Pública, 95, e202101015.

\section{CITAR ESTE ARTICULO:}

Villacis Pérez, B. A., Camacho Martínez, D. S., Narváez Cevallos, F. E., \& Adriana Belén, C. M. (2021). Consumo de alcohol durante la pandemia COVID-19 en América Latina y el Caribe. RECIAMUC, 5(4), 232-244. https://doi. org/10.26820/reciamuc/5.(4).noviembre.2021.232-244

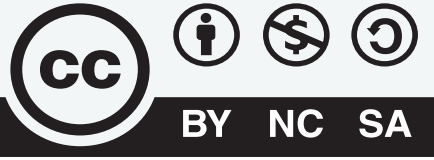

CREATIVE COMMONS RECONOCIMIENTO-NOCOMERCIAL-COMPARTIRIGUAL 4.0. 